\title{
Pyogenic liver abscess and pulmonary septic emboli in a diabetic woman
}

\author{
Aloísio Felipe-Silva ${ }^{a}$, Lorena Laborda ${ }^{b}$
}

Felipe-Silva A, Laborda L. Pyogenic liver abscess and pulmonary septic emboli in a diabetic woman. Autopsy Case Rep [Internet]. 2011;1(1):33-41.

\section{ABSTRACT}

A 67-year-old diabetic woman was admitted with abdominal pain one day after being discharged from other hospital, where she had been diagnosed with urinary infection and sepsis. A pyogenic liver abscess was detected by ultrasonography in this institution. Antibiotic therapy was started and a percutaneous drainage was attempted, but major clinical deterioration with irresponsive septic shock and acidosis ensued. Autopsy confirmed pyogenic liver abscess and findings of septic shock. Rarely documented microscopic septic pulmonary emboli were detected. This case illustrates the importance and difficulty of early recognition of pyogenic liver abscess and its severe septic complications in diabetic patients. Microscopic septic pulmonary embolism is a rare finding in pyogenic liver abscess cases and its precise clinical significance is unknown.

Keywords: Autopsy; Liver abscess; Pulmonary embolism; Diabetes Mellitus; Sepsis

\section{CASE REPORT}

A 67-year-old Brazilian female was referred to this service with abdominal pain. On admission in the emergency room she complained of right lower hemithorax pain, abdominal volume increase, weight loss (6kg in a month) and malaise. She also had a couple of vomiting episodes in the last day. She had type 2 diabetes mellitus (diagnosed at age $57)$ and was a cigarette smoker.

She had been hospitalized for the last 2 weeks in another institution because of decompensated diabetes, sepsis from urinary infection and anemia. She was discharged from that institution the day before she was admitted in this hospital.
She had been taking metformin and oral norfloxacin since the first hospitalization.

Physical examination revealed moderate mucosal pallor, moderate abdominal distention, and an enlarged and painful liver. Moderate edema in lower limbs was noted. Heart rate was $97 \mathrm{bpm}$ and blood pressure was $80 / 50 \mathrm{mmHg}$. Temperature was $37^{\circ} \mathrm{C}$. Respiratory rate and neurological status were normal.

Laboratory tests on admission are shown in table 1. Urine culture was negative.

\footnotetext{
${ }^{a}$ Anatomic Pathology Service - Hospital Universitário - Universidade de São Paulo, São Paulo/SP - Brazil.

b Department of Internal Medicine - Hospital Universitário - Universidade de São Paulo, São Paulo/SP - Brazil.
} 
Table 1 - Laboratory data on admission .

(Abnormal values are highlighted)

\begin{tabular}{|c|c|}
\hline Albumin (g/dL) & 2.5 \\
\hline Hemoblobin (g\%) & 6.3 \\
\hline Hematocrit (\%) & 19 \\
\hline $\mathrm{MCV}(\mathrm{fL})$ & 80 \\
\hline $\mathrm{MCH}(\mathrm{pg})$ & 27 \\
\hline Leukocytes $/ \mathrm{mm}^{3}$ & 2700 \\
\hline Band neutrophil (\%) & 1 \\
\hline Segmented neutrophil (\%) & 92 \\
\hline Eosinophils (\%) & 1 \\
\hline Lymphocytes (\%) & 4 \\
\hline Monocytes (\%) & 2 \\
\hline Platellets $/ \mathrm{mm}^{3}$ & 261.000 \\
\hline GOT/AST (U/L) & 50 \\
\hline GPT/ALT (U/L) & 30 \\
\hline Total bilirubin (mg/dL) & 0.3 \\
\hline Direct bilirubin $(\mathrm{mg} / \mathrm{dL})$ & 0.2 \\
\hline Potassium (mEq/L) & 3.2 \\
\hline Sodium $(\mathrm{mEq} / \mathrm{L})$ & 139 \\
\hline Creatinine (mg/dL) & 1.6 \\
\hline Urea (mg/dL) & 84 \\
\hline Glycemia (mg/dL) & 153 \\
\hline APTT (s) & 57 \\
\hline INR & 1.28 \\
\hline
\end{tabular}


Abdominal ultrasound revealed an enlarged liver and a $15.8 \mathrm{~cm}$ irregularly contoured septated cystic lesion in the right lobe, with some gas content. These findings were consistent with a liver abscess. Right kidney was not visualized. Other abdominal organs were normal. Supportive measures, erythrocyte concentrate transfusion and antibiotic therapy with ceftriaxone and metronidazole were started.

CT imaging confirmed a large and ill-delimited hypodense mass that occupied almost the entire right hepatic lobe. Atrophy of the right kidney with caliceal and right ureteral calculi and a small cyst in left kidney were the only other findings.

A diagnostic US-guided percutaneous needle aspiration detected purulent material. Microbiological cultures were all negative (aerobic, mycobacteria and fungi). Rare gram-negative bacilli were detected on direct Gram staining. A diagnosis of sepsis secondary to pyogenic liver abscess was established.

The patient was considered non-eligible for open surgical treatment at that moment because of poor clinical and laboratory parameters. A percutaneous drainage was attempted and a large amount of purulent and hemorrhagic material was drained.
About one hour after needle aspiration, the patient presented the first episode of cardiac arrest (pulseless electrical activity) which was reverted in 2 min with standard cardiopulmonary resuscitation procedures. Under intensive care the patient presented major clinical deterioration due to irresponsive septic shock and acidosis. She had 6 further episodes of cardiac arrest within the following 6 hours and died. Total time of hospitalization was 2 days.

\section{Autopsy}

The liver weighted $3336 \mathrm{~g}$ (normal $1500 \mathrm{~g}$ ). Grossly there was a $15 \mathrm{~cm}$ encapsulated and multiloculated collection, filled with whitish-yellow material (Figure 1). Surrounding liver was markedly congested. The cutaneous and hepatic sites of drainage were unremarkable. No hemorrhagic collections were seen. There was no biliary tract tumor or calculi. Histological examination revealed an organizing abscess filled with reactive histiocytes, neutrophils, fibrin and necrotic debris (Figure 2). Rare gram-positive bacilli were detected by BrownBrenn stain (Figure 3). Stains for fungi and protozoan were negative.

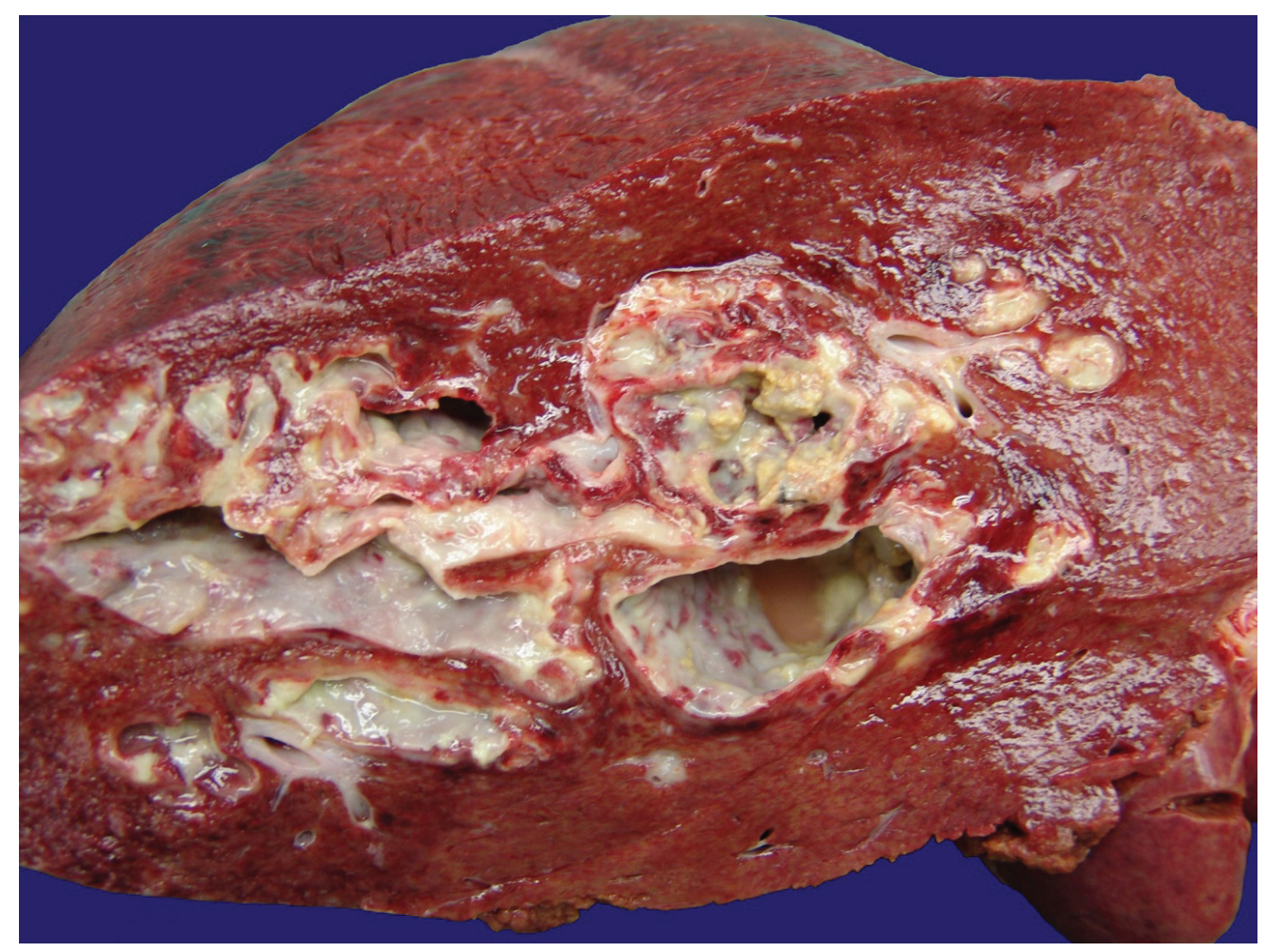

Figure 1- Multiloculated pyogenic liver abscess (close view, gross) 




Figure 2- Abscess content with histiocytes, neutrophils, fibrin and necrotic debris (H\&E, 400x)

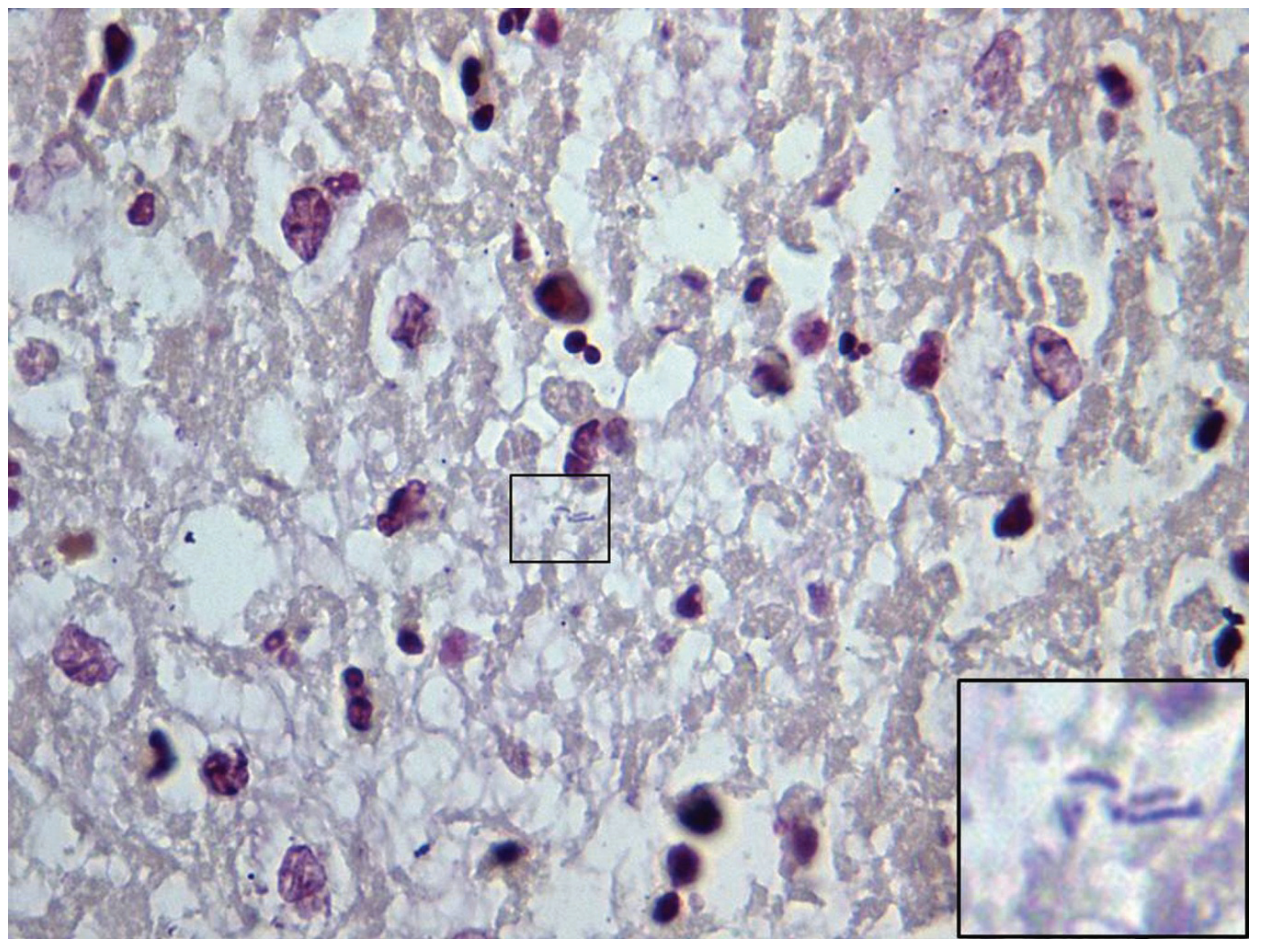

Figure 3- Rare gram-positive bacilli (bluish) in liver abscess (Brown-Brenn stain, 1000x - oil immersion) 
The right and left lungs were heavier than normal (615 and $545 \mathrm{~g}$ respectively; normal range $360-570$ and $325-480 \mathrm{~g}$ ), congested and lightly edematous. Microscopic obstructive foci of necrotic debris and fibrin similar to abscess content were de- tected in small lung artery branches. Round and optically clear spaces consistent with gas bubbles could be seen (Figure 4). These findings were interpreted as embolization of liver abscess content to the lungs (septic embolism).

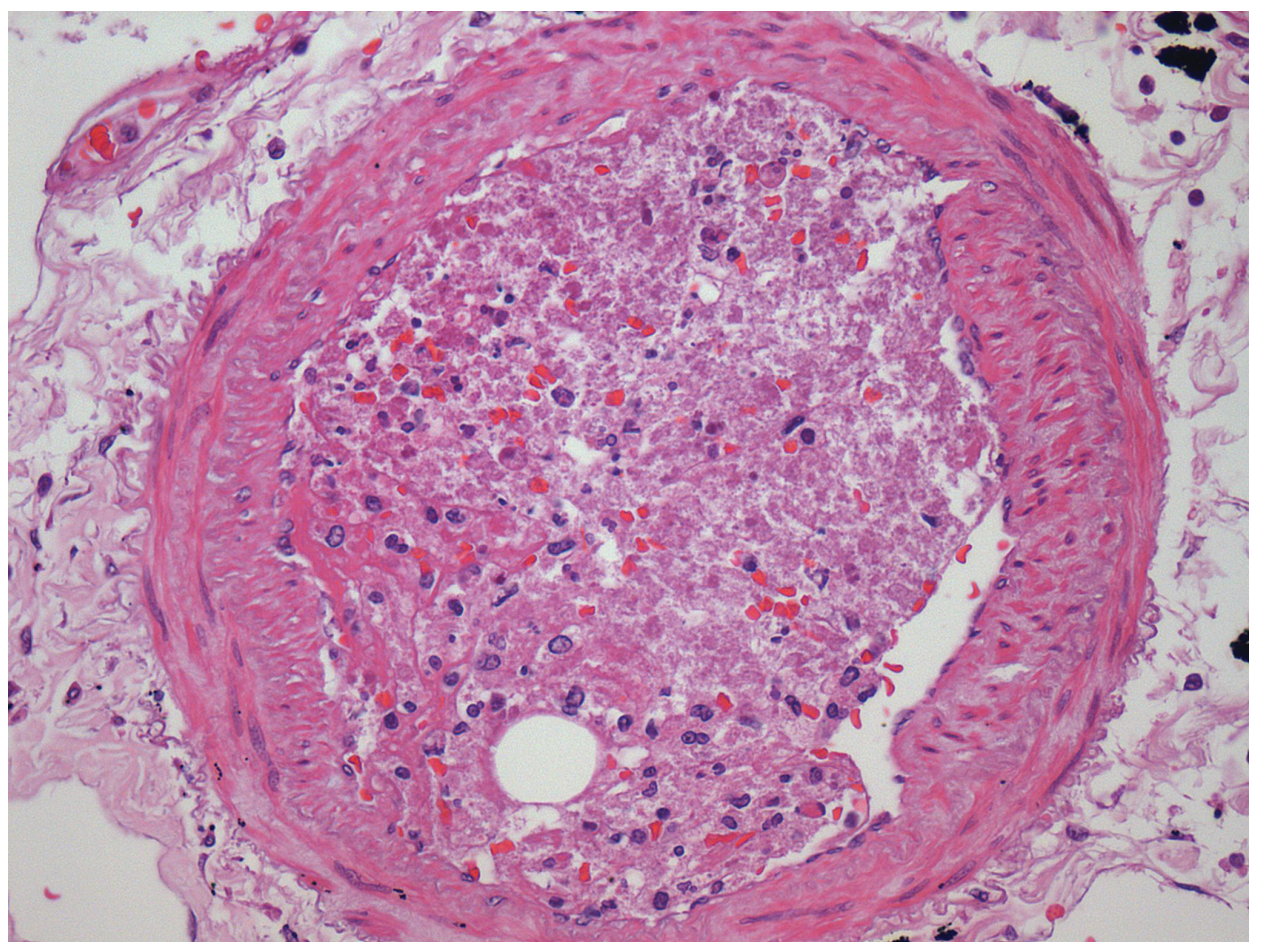

Figure 4- Septic embolization from liver abscess in lung arteriole. Note round optically clear space in the bottom (gas bubble). (H\&E, 400x)

Systemic findings of sepsis and shock were observed in other organs: red neurons in central nervous system; lipid depletion and hemorrhagic foci in adrenal glands. Severe reactive erythrophagocytosis (Figure 5) and other reactive changes were seen in spleen, lymph nodes and bone marrow.

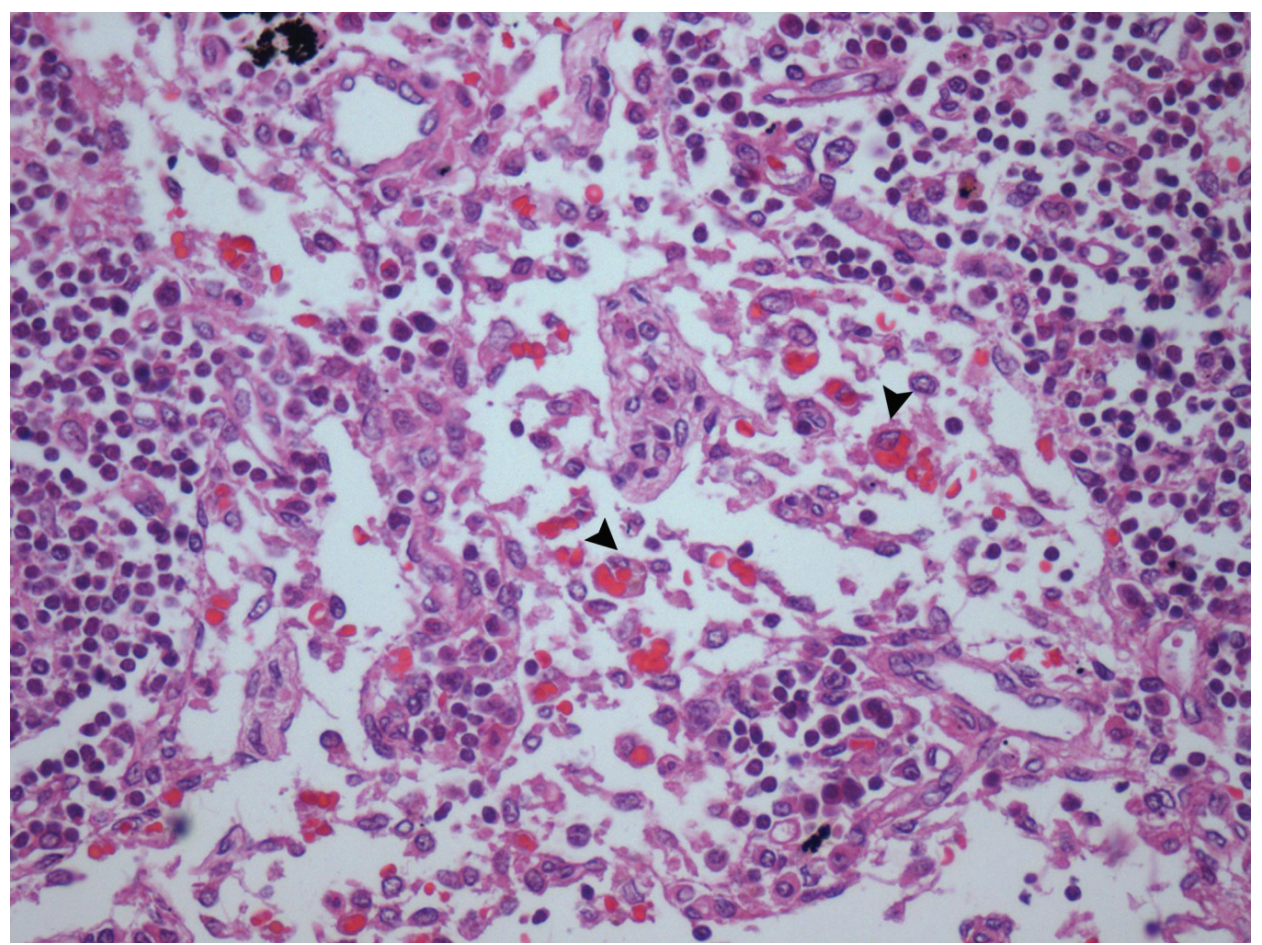

Figure 5- Severe erythrophagocytosis in lymphnode (arrowheads) (H\&E, 400x) 
Diabetic hyaline arteriolosclerosis was detected in kidneys, spleen and pancreas (Figure 6). Left kidney was atrophic with calculi and chronic pyelonephritis (Figure 7). Other relevant autopsy findings were severe atherosclerosis of aorta and coronaries, and pulmonary changes related to cigarette smoking (respiratory bronchiolitis, anthracosis and apical emphysema).

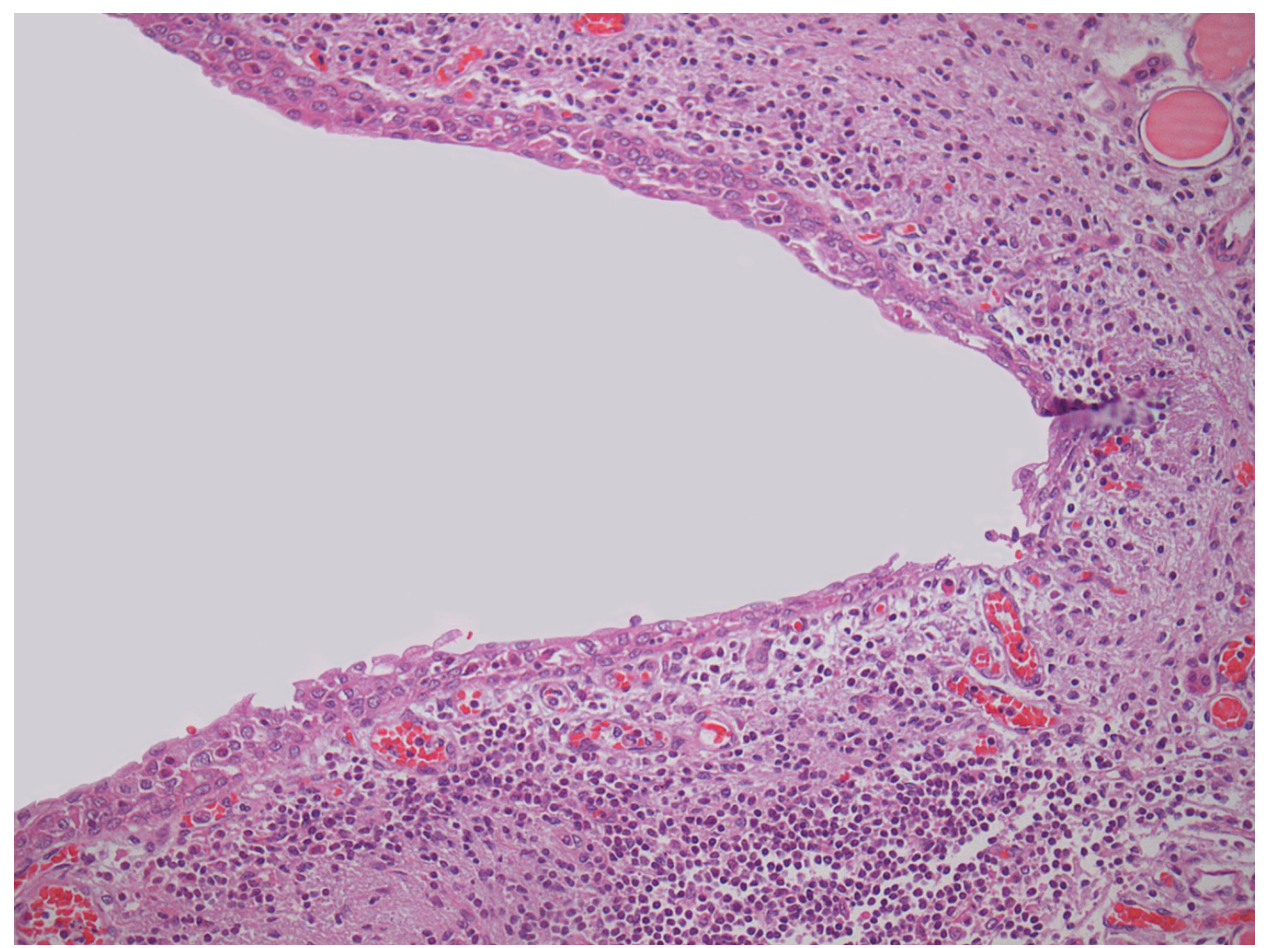

Figure 6 - Chronic pyelonephritis with dense mixed inflammatory infiltrate in renal pelvis (H\&E, 400x)

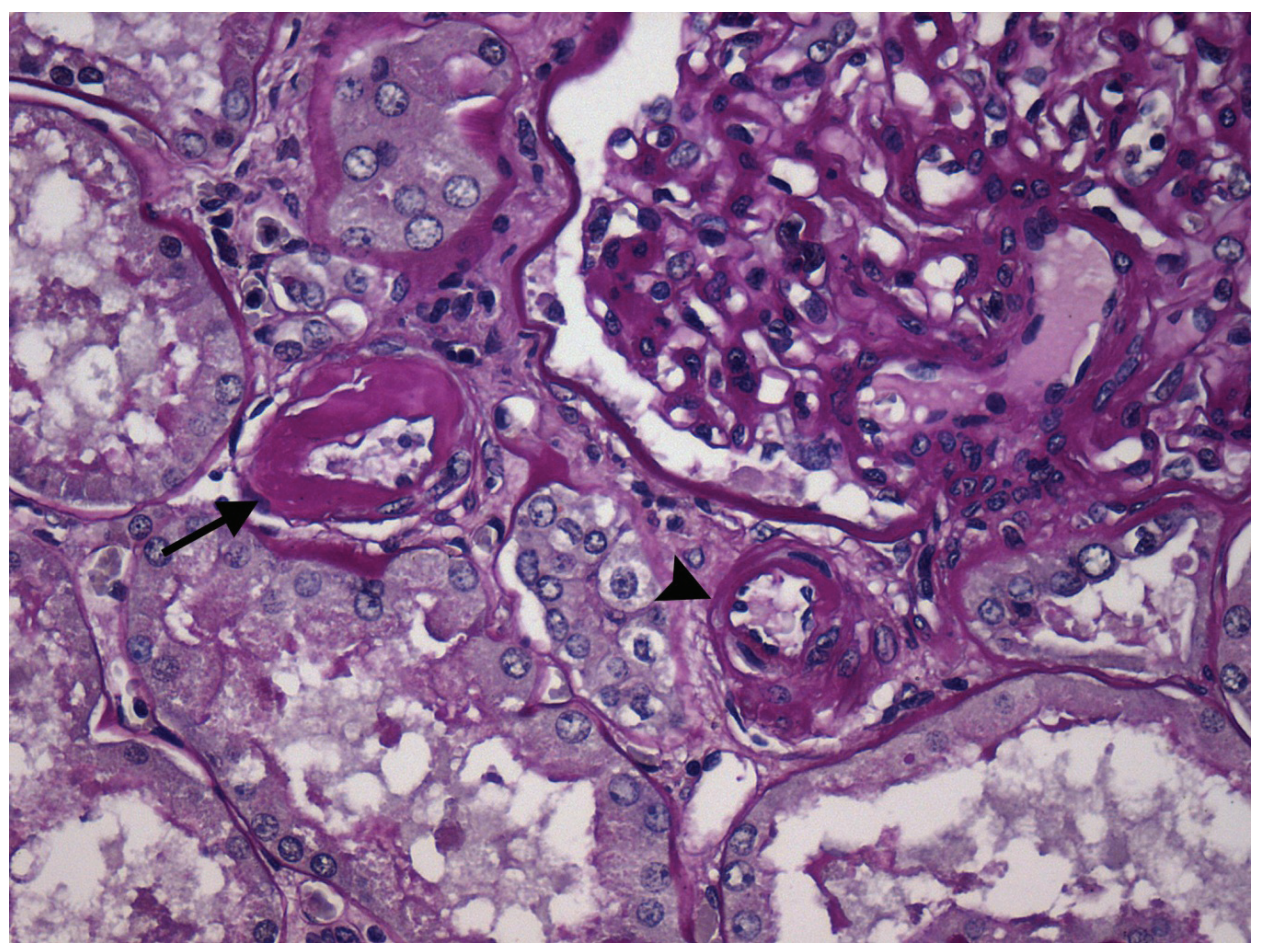

Figure 7 - Chronic pyelonephritis with dense mixed inflammatory infiltrate in renal pelvis (H\&E, 400x) 


\section{DISCUSSION}

We report a case of lethal septic complications from pyogenic liver abscess (PLA) in a diabetic woman. Autopsy confirmed major clinical impressions and also revealed rarely documented microscopic septic pulmonary emboli.

Liver abscesses are usually classified as pyogenic or amoebic. PLA is an infrequent and often severe disease. Important predictors of mortality are gas-forming liver abscess, poor renal function and high Acute Physiology and Chronic Health disease Classification System II (APACHE II) score on admission. Age and patient's underlying pathology may play a hole as well. ${ }^{1,2,3}$

There has been a decrease in mortality rates in the last decades due to improvement in diagnosis, with imaging techniques, and treatment, with the improvement of antimicrobial therapy and the development of percutaneous drainage. ${ }^{4}$

Early PLA symptoms are typically nonspecific and insidious, evolving from days to weeks. More specific symptoms include fever, abdominal pain (usually in the right upper quadrant), chills, anorexia and weight loss (usually in long standing PLA). Cough and pleuritic chest pain are less common. Physical examination may be normal or detect hepatomegaly, abdominal tenderness, a palpable mass or jaundice. Septic shock complicates delayed diagnosis. ${ }^{4,15}$

PLA may be caused by dissemination of infection from the abdominal cavity (via portal system), from systemic sites (via arterial system) or directly extension (either from biliary tree or traumatic). A predisposing condition is not identified in about half of the patients (cryptogenic PLA); however, in autopsy series this rate may be as low as $6 \%{ }^{5}$

Several studies have identified gastrointestinal sources or underlying biliary tract disease in up to half of the patients with pyogenic liver abscess. These causes included biliary disease, gastrointestinal cancer, pancreatitis, surgery and appendicitis. ${ }^{6,7,8,9}$

Diabetes, alcoholism, liver transplantation and malignancies are predisposing immunosuppressive states.4,10. Diabetes is a strong risk factor for PLA ${ }^{11}$ as well as for the development of metastatic infection from a PLA. ${ }^{12}$ The prevalence of diabetes in patients with PLA ranges from $10-27 \%$ up to $67,5 \%$, as reported in Taiwan. ${ }^{13}$
PLA are often polymicrobial and common isolated microorganisms are Escherichia coli, Klebsiella pneumoniae, Staphylococcus sp, Streptococcus $\mathrm{sp}$ and Bacteroides $\mathrm{sp} .{ }^{14,15}$ However, some studies have commonly found a single causative microorganism. ${ }^{9,13}$ Klebsiella infections seem to occur more commonly in the Asian population and are increasingly found USA ${ }^{16,17}$. E. coli seems to be a common pathogen in Brazil. ${ }^{15}$ Abscess and blood cultures are positive in $47-95 \%$ and $25-65 \%$ of the cases, respectively. ${ }^{14,15,18,19}$ Rare gram-negative and gram-positive bacilli were detected in the present case.

Septic pulmonary embolism (SPE) is characterized by the presence of an infected embolus that may lead to lung infarction and local abscess. It is a well-recognized and uncommon complication of intravenous drug use, right-side bacterial endocarditis, pelvic thrombophlebitis, prolonged indwelling catheter, arteriovenous shunts for hemodialysis, suppurative processes in the head and neck soft tissues and gangrene of extremities. ${ }^{20,21}$ Clinical presentation is generally nonspecific. Symptoms are insidious with fever, cough, hemoptysis, or complicated by lung abscess, empyema and massive hemoptysis. ${ }^{22}$

SPE has been described as a rare septic complication of PLA. In a large autopsy series from Japan, liver abscess was the primary focus of infection in $4.0 \%$ of "critical" SPE cases. ${ }^{23}$ Other metastatic complications of PLA include endophthalmitis, cerebral abscesses, purulent meningitis, otitis media, osteomyelitis, arthritis, prostate abscesses, pylephlebitis and psoas muscle abscesses. ${ }^{16} \mathrm{He}-$ patic veins thrombophlebitis may be related to lung dissemination. ${ }^{24}$ Chest computed tomography scan may be useful for this diagnosis in diabetic patients with PLA and suspicious chest radiograph findings. ${ }^{21,22}$

This patient had complicated diabetes as a major risk factor for PLA and hematogenous spread to the lungs. Retrospective calculated APACHE II score was 18 on admission. APACHE II scores $\geq 15$ have been associated with higher mortality rates. ${ }^{1,25}$ Clinical and pathological evidence suggests that the urinary tract was the primary source of infection, although specific causative organisms could not be isolated. In Gaskin et al autopsy series of PLA cases, primary infection of the urinary tract was detected in $6 \%$ of adult patients. ${ }^{5}$ No septic emboli or infectious foci were identified in other organs in the present case. 
Curiously this patient was not febrile during hospitalization. Fever has been reported as the most common symptom in PLA (89.6 - 100\%), usually followed by right upper quadrant abdominal pain (58.8 - $72.2 \%)$ and chills $(35-69 \%)^{9,15}$ Age, diabetes and previous antimicrobial therapy may have contributed to this uncommon clinical presentation. This patient was already anemic at admission. Anemia was likely caused by chronic septic condition, poor nutritional state and presumed chronic renal failure caused by diabetic nephropathy. Autopsy ruled out hemorrhagic complications and iatrogenic bleeding. Severe erythrophagocytosis which is usually related to sepsis and transfusions might have contributed as well. The severe acute infection might also explain the lack of leukocytosis (actually the patient was leukopenic) since the demand for neutrophils in the large abscess can result in a loss of the systemic storage pool of neutrophils. Hypoalbuminemia is a common finding according to a large study. ${ }^{9}$ There was no clinical, laboratorial or pathological evidence of disseminated intravascular coagulation.

This patient had a diagnosis of urinary sepsis and she had a prescription of norfloxacin, a second generation quinolone with good spectrum for Gram negative rods. However, norfloxacin has low bioavailability, about $30-40 \%$, and low tissue concentration, which makes this drug a second-line choice to treat a severe infection like urinary sepsis ${ }^{26}$. Previous use of antibiotics may also have been a risk factor for the emergence of multidrug-resistant

\section{REFERENCES}

1. Chen SC, Tsai SJ, Chen CH, Huang CC, Lin DB, Wang $\mathrm{PH}$, et al. Predictors of mortality in patients with pyogenic liver abscess. Neth J Med. 2008 May;66(5):196-203.

2. Chen SC, Lee YT, Yen CH, Lai KC, Jeng LB, Lin DB, et al. Pyogenic liver abscess in the elderly: clinical features, outcomes and prognostic factors. Age Ageing. 2009 May;38(3):271-6; discussion.

3. Chen W, Chen $\mathrm{CH}$, Chiu KL, Lai HC, Liao KF, Ho YJ, et al. Clinical outcome and prognostic factors of patients with pyogenic liver abscess requiring intensive care. Crit Care Med. 2008 Apr;36(4):1184-8.

4. Reid-Lombardo KM, Khan S, Sclabas G. Hepatic cysts and liver abscess. Surg Clin North Am. 2010 Aug;90(4):679-97.

5. Gaskin DA, Bodonaik NC, Williams NP. Hepatic abscesses at the University Hospital of the West Indies. A 24-year autopsy review. West Indian Med J. 2003 Mar;52(1):37-40. strains, which could be resistant to the treatment used in this case. This patient received standard of care antimicrobial therapy according to most literature and expert opinions.

It is interesting to note that SPE was detected at histological examination with no other major inflammatory or vascular findings in the lungs. This may be explained by factors like the size of the embolus and of the occluded vessel, the amount of microbes and the pulmonary double circulation system. These data suggest that microscopic SPE were more likely terminal and not decisive in the cause of death. On the other hand, it could be hypothesized that these microscopic septic emboli might be an initial manifestation of mechanical septic embolization. Whether microscopic SPE was part of septic complications or related to diagnostic and therapeutic procedures is rather speculative.

In summary, this autopsy case illustrates the importance and difficulty of early recognition of PLA and its severe septic complications in diabetic patients. Microscopic SPE is a rare finding in PLA cases and its precise clinical significance is unknown.

\section{Acknowledgments:}

We are grateful to Rosa Maria Conceição Zanardi and Júlio César de Santana for the technical support on the visual work.

6. Alvarez Pérez JA, González JJ, Baldonedo RF, Sanz L, Carreño G, Junco A, et al. Clinical course, treatment, and multivariate analysis of risk factors for pyogenic liver abscess. Am J Surg. 2001 Feb;181(2):177-86.

7. Seeto RK, Rockey DC. Pyogenic liver abscess. Changes in etiology, management, and outcome. Medicine (Baltimore). 1996 Mar;75(2):99-113.

8. Bissada AA, Bateman J. Pyogenic liver abscess: a 7-year experience in a large community hospital. Hepatogastroenterology. 1991 Aug;38(4):317-20.

9. Rahimian J, Wilson T, Oram V, Holzman RS. Pyogenic liver abscess: recent trends in etiology and mortality. Clin Infect Dis. 2004 Dec;39(11):1654-9.

10. Kaplan GG, Gregson DB, Laupland KB. Population-based study of the epidemiology of and the risk factors for pyogenic liver abscess. Clin Gastroenterol Hepatol. 2004 Nov;2(11):1032-8.

11. Thomsen RW, Jepsen P, Sørensen HT. Diabetes mellitus and pyogenic liver abscess: risk and prognosis. Clin In- 
fect Dis. 2007 May;44(9):1194-201.

12. Chen SC, Lee YT, Lai KC, Cheng KS, Jeng LB, Wu WY, et al. Risk factors for developing metastatic infection from pyogenic liver abscesses. Swiss Med Wkly. 2006 Feb;136(7-8):119-26.

13. Wang JH, Liu YC, Lee SS, Yen MY, Chen YS, Wann SR, et al. Primary liver abscess due to Klebsiella pneumoniae in Taiwan. Clin Infect Dis. 1998 Jun;26(6):1434-8.

14. Branum GD, Tyson GS, Branum MA, Meyers WC. Hepatic abscess. Changes in etiology, diagnosis, and management. Ann Surg. 1990 Dec;212(6):655-62..

15. Herman P, Pugliese V, Salem MZ, Machado MA, Bacchella T, da Cunha JE, et al. [Pyogenic hepatic abscess: report of 51 cases]. Rev Hosp Clin Fac Med Sao Paulo. 19941994 Nov-Dec;49(6):234-7. Portuguese.

16. Cheng DL, Liu YC, Yen MY, Liu CY, Wang RS. Septic metastatic lesions of pyogenic liver abscess. Their association with Klebsiella pneumoniae bacteremia in diabetic patients. Arch Intern Med. 1991 Aug;151(8):1557-9.

17. Pastagia M, Arumugam V. Klebsiella pneumoniae liver abscesses in a public hospital in Queens, New York. Travel Med Infect Dis. 2008 Jul;6(4):228-33.

18. Mezhir JJ, Fong Y, Jacks LM, Getrajdman GI, Brody LA, Covey AM, et al. Current management of pyogenic liver abscess: surgery is now second-line treatment. J Am Coll Surg. 2010 Jun;210(6):975-83.

19. Andrés FS, Nicolás AA, Antonia VV, Nivia EA, Ariel CL. Absceso hepático: Serie de 107 casos y revisión de la literatura. Rev. chil. infectol. 2009 Feb;26(1): 49-53. Spanish.
20. Cook RJ, Ashton RW, Aughenbaugh GL, Ryu JH. Septic pulmonary embolism: presenting features and clinical course of 14 patients. Chest. 2005 Jul;128(1):162-6.

21. Zenda T, Araki I, Hiraiwa $Y$, Miyayama S, Masunaga T, Takeda Y, et al. Septic pulmonary emboli secondary to pyogenic liver abscess in a diabetic patient. Intern Med. 1995 Jan;34(1):42-5.

22. Yang PW, Lin HD, Wang LM. Pyogenic liver abscess associated with septic pulmonary embolism. J Chin Med Assoc. 2008 Sep;71(9):442-7.

23. Sakuma M, Sugimura K, Nakamura M, Takahashi T, Kitamukai O, Yazu T, et al. Unusual pulmonary embolism: septic pulmonary embolism and amniotic fluid embolism. Circ J. 2007 May;71(5):772-5.

24. Maffiolo C, Novellas S, Chevallier P, Brunner P, Mourou MY, Bruneton JN. Thrombophlebitis of the hepatic veins: complication of a Klebsiella liver abscess. Clin Imaging. 20062006 Jan-Feb;30(1):63-5.

25. Chen SC, Huang CC, Tsai SJ, Yen CH, Lin DB, Wang $\mathrm{PH}$, et al. Severity of disease as main predictor for mortality in patients with pyogenic liver abscess. Am J Surg. 2009 Aug;198(2):164-72.

26. Gupta K, Hooton TM, Naber KG, Wullt B, Colgan R, Miller LG, et al. International clinical practice guidelines for the treatment of acute uncomplicated cystitis and pyelonephritis in women: A 2010 update by the Infectious Diseases Society of America and the European Society for Microbiology and Infectious Diseases. Clin Infect Dis. 2011 Mar;52(5):e103-20.

\section{Conflict of interest: None}

Submitted on: $1^{\text {th }}$ February 2011

Accept on: 4th March 2011

Correspondence: Serviço de Anatomia Patológica

Aloísio Felipe-Silva

Av. Prof. Lineu Prestes, 2565 - Cidade Universitária - São Paulo - SP - Brazil

CEP: 05508-000 - Phone: +55.011. 3091.9379

E-mail: aloisiosilva@hu.usp.br 